\title{
Micellar polymerization of alkylammonium 2-acrylamido-2-methylpropane sulfonates in the solvents of different polarities and properties of resulting polyelectrolyte-surfactant complexes
}

\author{
A. Yu Bilibin - T. M. Shcherbinina - Yu. A. Kondratenko • \\ N. A. Zorina $\cdot$ I. M. Zorin
}

Received: 14 July 2014 / Revised: 9 December 2014 / Accepted: 28 December 2014 / Published online: 20 January 2015

(C) Springer-Verlag Berlin Heidelberg 2015

\begin{abstract}
Polymerization of formerly described novel surfmers polymerizable by counterion has been reported. Dodecylammonium, cetylammonium, and cetyltrimethylammonium 2-acrylamido-2-methylpropane sulfonates were polymerized in water-dioxane mixtures in a wide range of concentrations of the solvents and in some organic solvents as well. The resulting polyelectrolyte-surfactant complexes precipitated from water-dioxane mixtures. Molecular weights and polymerization yields of the products were the highest at high and low water contents in the reaction mixture, whereas the lowest polymerization degrees and yields conform to solutions, containing $20 \%$ of water. It has been found that the morphology of the precipitated aggregates formed in low-polarity solvents differs considerably from that formed in water. Polymerization in organic media depends strongly on the polarity of the solvent. Contributions of electrostatic and hydrophobic interactions in the structure formation of the complexes were estimated qualitatively.
\end{abstract}

Keywords Counterion surfmers · Micellar polymerization . Polyelectrolyte-surfactant complexes · Precipitation ·

Morphology

\section{Introduction}

Ionic complexes of polyelectrolytes and oppositely charged surfactants (polyelectrolyte-surfactant complex

A. Y. Bilibin $(\varangle) \cdot$ T. M. Shcherbinina • Y. A. Kondratenko •

N. A. Zorina $\cdot$ I. M. Zorin

Department of Chemistry, Saint Petersburg State University,

198504University Av., 26, Saint Petersburg, Russia

e-mail: alex_bilibin@mail.ru
(PSC)) attract much attention during the past decades due to their ability to form nanostructured systems, their biological relevance, and other specific features [1-6]. They are of interest because of their fundamental and practical importance. PSCs are used in drug delivery systems [7], preparation of nanomaterials [8], cosmetics, foods, paints [9], solar cells [10], and so on [11, 12].

Usually, PSCs are prepared by direct mixing of water solutions of a polyelectrolyte and an oppositely charged surfactant [2]. Thus, there are ions of four types in the reaction mixture. The complex should be purified from an excess of the surfactant and additional ions using rather a complicated procedure. Both stoichiometric and non-stoichiometric PSC can be prepared in this route. The binding is highly cooperative as a rule [13]. It means that the binding of the first surfactant molecule at a certain position of the polyelectrolyte chain makes the adjacent ionic groups much favorable for binding other surfactant molecules. Thus, binding proceeds according to zipper mechanism. The resulting stoichiometric PSC precipitates from the solution as a rule.

"Insoluble polyelectrolyte-surfactant complexes display a myriad of structures" [14]. Water-insoluble PSCs really form a great diversity of structures because of a difference in a nature of ions, polyelectrolyte chain rigidity, and length and volume of the hydrophobic part of the surfactant molecule. Moreover, the same sample of PSC can form different internal structures depending on mixing and precipitation conditions.

Morphology and structure of the precipitated phases depend on very many parameters, such as a nature of both polyelectrolyte and surfactant, concentration of the solutions, and even on a sequence of adding the components. Formation of an internal structure of PSC is associated with both hydrophobic and electrostatic attractive interactions. Hydrophobic interactions are proportional to a portion of hydrophobic 
elements in the complex (hydrophobic-hydrophilic balance). Electrostatic interactions are associated mainly with a nature of ionic group of both surfactant and polyelectrolyte and average linear charge density of the polyelectrolyte $\xi$

$$
\xi=\frac{e^{2}}{4 \pi \epsilon b k T}
$$

where $e$ is the protonic charge, $\epsilon$ is dielectric constant of the solvent, and $b$ is the distance between adjacent charges along the polymer backbone [15].

Many teams have been involved in the study of binding process and structure of the PSC formed by direct mixing of polyelectrolyte and oppositely charged surfactants with the use of different methods: DLS [15], SAXS and fluorescence $[16,17]$, surface tension [18], electronic microscopy [19], and many others. The authors [20] have performed computer simulation of phases and self-assembled structures of PSC depending on relative contributions of hydrophobic and electrostatic interactions in the structure formation.

Insolubility of PSC in water makes it difficult or impossible for some applications [21]. However, they have a great potential of application being processed from organic solvents $[2$, 4]. Piculell and co-authors [22] considered specific conditions for preparation of water-soluble complex salts of surfactant with polymeric (polycarboxylic) counterion which are of special interest as components for cosmetics. They have shown that the weaker the ionic groups of a polyelectrolyte and the lower its polymerization degree, the higher is solubility of the complex in water.

Some examples of water-soluble stoichiometric PSC prepared by polymerization of surfactants with polymerizable counterion have been reported in the literature. All publications dealing with polymerization of counterionpolymerizable surfmers were aimed at retention of the structure formed in the monomer solutions as a result of the polymerization.

Pileni et al. [23] polymerized cetyltrimethylammonium methacrylate in direct micelles, which retain their shape after polymerization. Phase separation did not occur, and no precipitate was formed. The product was neither isolated nor characterized.

Sanderson polymerized dodecylammonium acrylate and methacrylate forming lyotropic liquid crystalline phase in concentrated water solutions [24]. The authors reported that the conservation of the phases formed by monomers, bicontinuous cubic phase, always evolved to lamellar phase upon polymerization. The authors could not determine molecular masses of the polymerized samples, because of their insolubility in water and in all organic solvents.

Walker et al. have reported proper water-soluble polyelectrolyte-surfactant aggregate system prepared by micellar polymerization of cetyltrimethylammonium 4-vinylbenzoate. Walker's team is the only team which reported molecular characteristics of the polymerization products [25-27].

Recently, Shulevich et al. reported template polymerization of trimethyl-(methacryloyloxyethyl)ammonium methyl sulfate in a micellar solution of SDS to obtain water-soluble complexes enriched with the surfactant ions [28].

It should be noted that all cited publications dealt with polymerization of counterion surfmers composed of a cationic surfactant and rather weak polymerizable acids (acrylic, methacrylic, 4-vinylbenzoic acids).

Polymerization of surfmers consisting of strong acids has been studied much less. Cavicchi et al. described reversible addition-fragmentation chain transfer (RAFT) polymerization of trioctylammonium $p$-styrene sulfonate in benzene [29]. Later, they reported RAFT polymerization of $N, N$ dimethyloctadecylammonium $p$-styrene sulfonate in benzene and study the gelation of resulting PSC in low-polarity organic solvents [30]. In both cases, polymerization proceeded in molecular-dispersed solutions. Micelle-forming properties of the monomers have not been discussed.

Our team has published preliminary data on precipitation polymerization of novel counterion surfmerdodecylammonium 2-acrylamido-2-methylpropane sulfonate (DDA-AMPS) - in solvents of different polarities [31]. We consider this polymerization as an alternative route to preparation of strictly stoichiometric water-insoluble PSC, which should possess specific nanostructure determined by the synthesis conditions. In the present work, we report new results on polymerization DDA-AMPS and two novel counterion surfmers: cetylammonium 2-acrylamido-2-methylpropane sulfonate (CA-AMPS) and cetyltrimethylammonium 2acrylamido-2-methylpropane sulfonate (CTA-AMPS) whose synthesis and micelle-forming properties have been described in [32]. It may be expected that the structures and morphologies of the water-insoluble PSC prepared in such a route should differ significantly from those of PSC prepared in traditional manner. The main aim of the work was to study the polymerization of the surfmers in water-dioxane mixtures with different ratios of the solvent components and the variation of polarity of the system as a result and to follow the changes, which occur during transformation of the system from micellar monomeric to solid-state PSC. Besides, some polymerizations of the surfmers were performed in organic solvents. As to our knowledge, polymerization of these and analogous surfmers in solvents with different polarities has not been published yet.

\section{Materials and methods}

Synthesis and properties of DDA-AMPS, CA-AMPS, and CTA-AMPS have been described in [32]. Ammonium 
persulfate (APS), azobisisobutyronitrile (AIBN), and 4,4'azobis(4-cyanovaleric acid) (ACVA) were purchased from Merck and used as received. Freshly distilled or deionized water with conductivity less than $2 \mu \mathrm{S} / \mathrm{cm}$ was used for all preparations. Organic solvents (dioxane, benzene, toluene, xylene, methylene chloride, chloroform, tetrachloromethane, tetrahydrofurane, $\mathrm{N}, \mathrm{N}$-dimethylformamide) were purified according standard methods of purification.

${ }^{1} \mathrm{H}$ NMR spectra were recorded using Bruker DPX-300 at $300 \mathrm{MHz}$ in $\mathrm{CDCl}_{3}$ and DMSO- $\mathrm{d}_{6}$. AFM experiments were performed using Veeco Nanoscope V (tapping mode).

Scanning electron microscopy investigations were performed with a Zeiss Supra 40VP instrument at an accelerating voltage of $2 \mathrm{kV}$.

Viscosity measurements of the reaction mixture during the process of polymerization were taken on automated vibration viscometer SV-10a (A\&D, Japan), equipped with $12 \mathrm{~mL}$ volume of thermostated cell.

Conductometry was performed at polymerization temperature using an "Expert 002" conductometer (Econics Expert Ltd., Russia) with a filler-type sensor (measurement range $0.01-1999 \mu \mathrm{S} / \mathrm{cm})$.

Ubbelohde viscometer (capillary diameter $0.22 \mathrm{~mm}$ ) was used for viscosity measuring of the polymer solutions in organic solvents.

\section{Polymerization of the surfmers}

The polymerization was conducted in the mixtures with dioxane content in the range of $0-100 \%$ at $60{ }^{\circ} \mathrm{C}$ with APS as initiator in water-enriched mixtures and ACVA in dioxaneenriched mixtures. The polymers precipitated at initial stages of the process in all cases. The products were filtered off, washed by water and acetone, and dried to the constant weight. The polymerization processes and the products were characterized by yields and intrinsic viscosity values (chloroform, $25{ }^{\circ} \mathrm{C}$ ).

Typical procedure for CA-AMPS polymerization in water and water-dioxane mixtures

CA-AMPS $(0.306 \mathrm{~g})$ was placed into a polymerization tube, and $2.7 \mathrm{~mL}$ of the solvent (dioxane-water mixture) was added $\left(C_{\mathrm{m}}=0.25 \mathrm{~mol} / \mathrm{L}\right)$. Dry argon was bubbled through the solution at permanent heating. In $10 \mathrm{~min}, 2.7 \mathrm{mg}$ of ammonium persulfate was added and the tube was placed in a thermostat. Polymerization was performed at $60{ }^{\circ} \mathrm{C}$ during $1 \mathrm{~h}$. Precipitation of the product started at the 10th min of the process. In $1 \mathrm{~h}$, the product was filtered off, washed with acetone, and dried. Yields and intrinsic viscosity values of the products are discussed in "Results and discussion" section (Fig. 3).
Typical procedure for CA-AMPS polymerization in organic solvents ( $p$-xylene)

CA-AMPS $(0.678 \mathrm{~g})$ was placed into a polymerization tube, and $11.9 \mathrm{~mL}$ of $p$-xylene was added $\left(C_{\mathrm{m}}=0.13 \mathrm{~mol} / \mathrm{L}\right)$. The content was heated up to dissolution of the monomer and bubbled with dry argon for $10 \mathrm{~min}$ at permanent heating; $23.7 \mathrm{mg}$ of AIBN was added, and the tube was placed in constant-temperature cuvette of a vibrational viscometer. The polymerization was performed at $75^{\circ} \mathrm{C}$ during $1.5 \mathrm{~h}$. Tough, transparent, opalescent gel formed as a result of the polymerization. It was crumbled up, washed with acetone, and dried, the yield was $0.540 \mathrm{~g}(80 \%)$.

${ }^{1} \mathrm{H}-\mathrm{NMR}$ spectra $\left(\mathrm{CDCl}_{3}\right)$ did not contain signals at 5.0 $6.5 \mathrm{ppm}$, which indicated that the product did not contain traces of the monomer.

\section{Results and discussion}

Polymerization of $\mathrm{N}$-alkylammonium 2-acrylamido-2methylpropyl sulfonates results in formation of polyelectrolyte-surfactant complexes (PSCs). As to our knowledge, salts of 2-acrylamido-2-methylpropyl sulfonic acid and cationic surfactants of primary and quaternary amines have not been studied as polymerizable surfactants.

Generally, PSC based on poly-2-acrylamido-2methylpropane sulfonate (PAMPS) and cationic surfactants can be obtained by direct mixing of water solutions of PAMPS (or its copolymers) and corresponding surfactant [18]. The stoichiometric complexes precipitate from water solutions, as a rule, but $100 \%$ binding of surfactants can be reached not in all cases. Moreover, the product should be purified of single ions, such as $\mathrm{Na}^{+}$and $\mathrm{Br}^{-}$. The purification process is rather complicated and laborious [2].

Polymerization of stoichiometric polymerizable salts should lead to formation of exactly stoichiometric complexes without an admixture of additional ions. Thus, it can be considered as a convenient and reliable route to preparation of exactly stoichiometric PSCs not contaminated with any other ions. Moreover, polymerization of the surfmers in different media can result in formation of products with new morphologies.

We have reported micelle-forming properties of three alkylammonium salts of AMPS in media with different polarities: dioxane-water mixtures, chloroform, $p$-xylene, and some other organic solvents [32]. AMPS-surfactant salts form direct micelles in water, molecular-dispersed solution in media of intermediate polarity, and reverse micelles in low-polarity solvents. The surfmers differ from each other by hydrophobicity and nature of ionic groups.

We have performed the polymerization in a number of solvent systems in order to study the effect of the monomers' 
phase state on the polymerization results (polymerization degrees, yields, microstructure, morphology, etc.).

Microstructure of the PSC obtained in traditional route depends strongly on preparation conditions: concentration of the components, sequence of mixing, and ratio of components. Thus, we suppose that the structure and morphology of the water-insoluble PSCs formed during polymerization of applicable surfmers can differ significantly from that of the PSCs prepared using the traditional route.

The samples of PSCs obtained by polymerization were investigated using a set of methods (AFM, SEM, NMR, WAXS).

Polymerization in water and water-dioxane mixtures

The majority of the results in the present work were obtained using water and water-dioxane mixtures as solvents for polymerization. All the surfmers are dissolved in these mixtures at a polymerization temperature. These systems were chosen as polymerization media because their polarity varies in wide range $\left(\epsilon=2.2\right.$ to 80 at $25^{\circ} \mathrm{C}$ ); thus, the formation of both direct and reverse micelles can be expected. Chemical structure and properties of the mixed solvent do not change substantially with change of the component ratio.

Polymerization of DDA-AMPS, CA-AMPS, and CTAAMPS proceeds with precipitation of the polymers in water and water-dioxane mixtures in a wide range of water-dioxane ratios. Thus, this polymerization is a particular case of precipitation polymerization [33], which begins in micellar solutions and transforms to precipitation polymerization in a few minutes after beginning of the process.

Precipitation polymerization attracts attention in the past decades due to its ability to produce particles of different sizes, shapes, and morphologies [34, 35]. Precipitation of a polymer on reaching of certain polymerization degree (critical oligomer degree of polymerization for precipitation, $j_{\text {crit }}$ ) [36] can be caused by different reasons: thermodynamic incompatibility, cross-linking, coil-to-glob transition, etc. Reasons of precipitation of the PSC will be discussed below.

Polymerization of neat vinyl chloride, acrylonitrile in water, and acrylic acid in supercritical carbon dioxide [37] is typical examples of the precipitation polymerization. The polymerization proceeds in the monomer absorbed within the precipitate as a rule. Termination reaction rate is very low because of a decrease of mobility of macroradicals. High molecular weight polymer samples can be obtained in this process. We have found no examples of precipitation polymerization, which begins in micellar solutions in the literature.

DDA-AMPS was the main object for studying main regularities of the process. Polymerization of DDA-AMPS in pure water solutions with a concentration of $8 \times 10^{-3} \mathrm{~mol} / \mathrm{L}$, which is below critical micelle concentration $(\mathrm{CMC})_{1}=1.1 \times$ $10^{-2} \mathrm{~mol} / \mathrm{L}$, proceeds without any appreciable changes of the reaction mixture during $1 \mathrm{~h}$. APS reacted with DDAAMPS, forming DDA persulfate, which precipitated and was insoluble in water at polymerization temperature $\left(60{ }^{\circ} \mathrm{C}\right)$. Thus, we used ACVA as the initiator in order that the solution remained homogenous. ${ }^{1} \mathrm{H}$ NMR spectra of the resulting product were similar to that of the monomer. Thus, the polymerization at a concentration below $\mathrm{CMC}_{1}$ did not occur, which conforms to literature data [38] and can be explained by very low monomer concentration.

Polymerization of alkylammonium AMPS salts in pure water at concentrations above $\mathrm{CMC}_{1}$ proceeds with high rates and ends less than $1 \mathrm{~h}$. In this case, no precipitate of dodecylammonium persulfate was formed at $60{ }^{\circ} \mathrm{C}$ on addition of APS. It means that persulfate anions react with DDA molecules aggregated in the micelles and become bound in a micelle adsorption layer.

The course of the polymerization process was followed up by conductometry. Generally, the conductivity of a solution decreases as the polymerization proceeds due to the reduction of mobility of charge carriers. Precipitation of the polymerization product should lead to more substantial decrease of conductivity.

Figure 1a represents the typical variation of conductivity of polymerization media on polymerization time. Increase of the conductivity during the initial step of the polymerization is associated with heating of the mixture; then, the inductive step follows, and after which, a decrease of conductivity starts (at $18 \mathrm{~min}$ ), which is a result of the polymerization in micellar solution. Abrupt decrease of the conductivity at $21 \mathrm{~min}$ corresponds to phase separation and formation of the precipitate.

Figure $1 \mathrm{~b}$ represents conductivity vs polymerization time plots for DDA-AMPS, CA-AMPS, and CTA-AMPS (the polymerization curve of AMPS-Na, proceeding without precipitation, is given for comparison). It would seem that the precipitation rates have to correlate with the hydrophobicity of the polymers formed. Thus, they should be in the following order: CTA-AMPS $>$ CA-AMPS $>$ DDA-AMPS. In fact, the precipitation rate of CTA-AMPS is the least, which can be attributed to its lowest polymerization rate due to the smaller size and lower counterion binding degree of their micelles [32]. This explanation well corresponds to the results of other experiments discussed below.

Figure 2 displays the variation of intrinsic viscosity of the products with initial concentration of the monomers in water solutions. It displays that polymerization degrees (and polymerization rates) of the products correspond to their precipitation rates. CTA-AMPS forms the products with the lowest polymerization degrees in the same conditions.

Polymerization of the surfmers in water-dioxane mixtures was performed to determine the influence of the solution structure on polymerization degrees of the products and their morphology. It has been found that the surfmers form direct micelles in the mixtures with water content above $20 \%$ and 


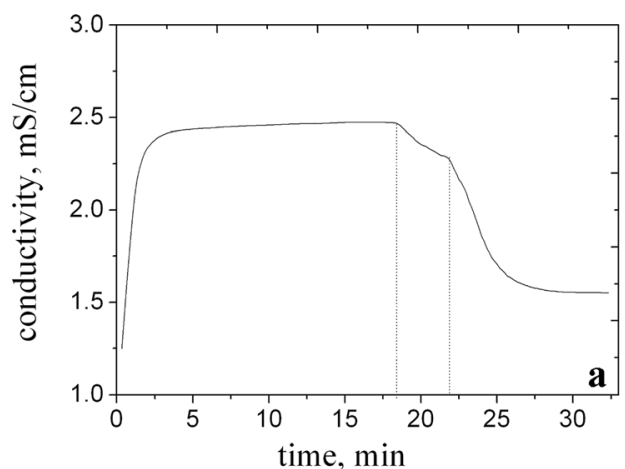

Fig. 1 a Dependence of conductivity of the polymerization media on polymerization time for polymerization of DDA-AMPS in water $(c=$ $0.1 \mathrm{M})$. b Dependence of normalized conductivity of the polymerization

reverse micelles in the mixtures with water content below $15 \% v / v[32]$. The polymerization was conducted with monomer concentrations in a range of $2.5 \times 10^{-1} \mathrm{~mol} / \mathrm{L}$ much above the $\mathrm{CMC}_{1}$ of the surfmers. Outwardly, it proceeds in the same manner as polymerization in pure water. All the polymers precipitated from the reaction mixtures at initial stages of the process. Figure 3 represents the dependence of the yields (Fig. 3a) and intrinsic viscosity values (Fig. 3b) of the polymers on water content in the solvent mixture.

Both plots have the same shape with minimum at water concentration about $20 \%$.

Thus, we assume that polymerization proceeds with high yields and high polymerization degrees of the products in both direct (above $20 \%$ of water content) and reverse (below $15 \%$ of water content) micellar solutions. It can be explained by the "effect of monomer condensation" [38], which leads to significant increase of local concentration of polymerizable groups either at the periphery of direct micelles or in the core of reverse micelles and increases polymerization rates as consequence.

The results received at studying of polymerization in water and water-dioxane mixtures give grounds to formulate a

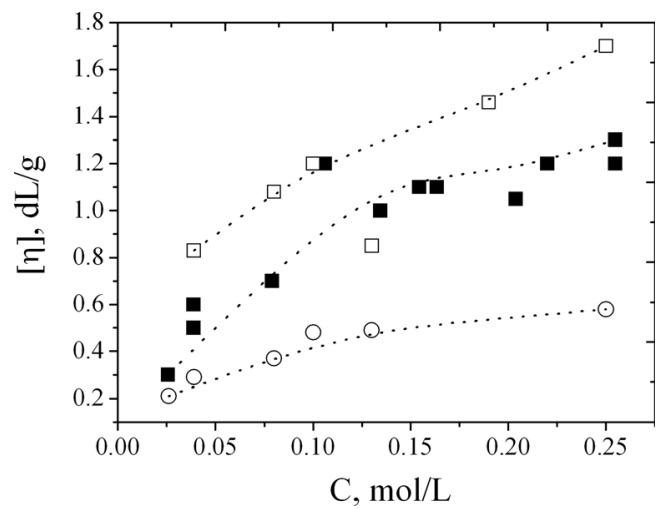

Fig. 2 Dependence of intrinsic viscosity of the products on initial concentrations of the monomers (empty square CA-AMPS, empty circle CTA-AMPS, filled square DDA-AMPS)

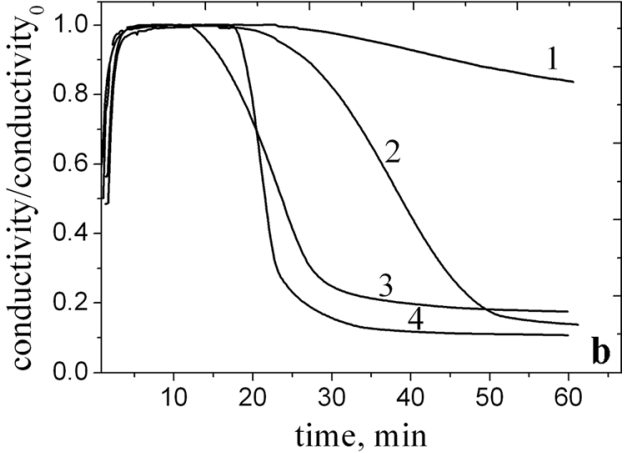

media on polymerization time for 1 AMPS-Na, 2 CTA-AMPS, 3 DDAAMPS, and 4 CA-AMPS

hypothesis of how the polymerization proceeds. At the very beginning of the polymerization in direct micelles at a concentration above $\mathrm{CMC}_{1}$, the most part of the surfmer molecules are located in the micelles. Only a small part of them remains in the molecular-dispersed solution as dissociated ions. Polymerizable AMPS-counterions are located predominantly in the micelles being either tightly bound with the micelle nucleus in compliance with binding degree value, or near the nucleus forming a diffuse layer, which surrounds the micelle as an ionic cloud whose density decreases with increasing distance from the nucleus.

Molecules of the initiators (both APS and ACVA) contain acidic moieties; thus, they should be located in the micelle too. Their binding degree should be even higher than that of AMPS due to their dibasic nature. Thereby, local concentration of both components of the polymerization is much higher in the micelle than their average overall concentration in the solution. It can be admitted that this concentration is near that in a neat monomer.

The most part of initiating free radicals are formed in the diffuse layer of micelles and initiate neighboring AMPS ions immediately. At the initial step of the polymerization, all the events take place in the adsorbed and diffuse layers at the surface of the micelle where both polymerizable and initiating anions are located. As the polymerization proceeds, proper ionic pairs of micelles transform in tight ionic pairs, "wrapped" in hydrophobic shell. Binding degree grows up to 1.0 instead of 0.6 in the micelles. This leads to disruption of micelles and formation of initial precipitate nuclei.

Initially free oligoradicals of AMPS, interacting each other and with micelle surfactant cations, destroy the micelle and form the starting macromolecules of PSC. At the main and the final stages of the polymerization, adjacent PSC chains aggregate each other to form nucleation centers and phase separation occurs. The monomer is concentrated at the surface of the precipitated polymer particles. Polymerization proceeds in the adsorbed layers of the monomer. Termination rate is 


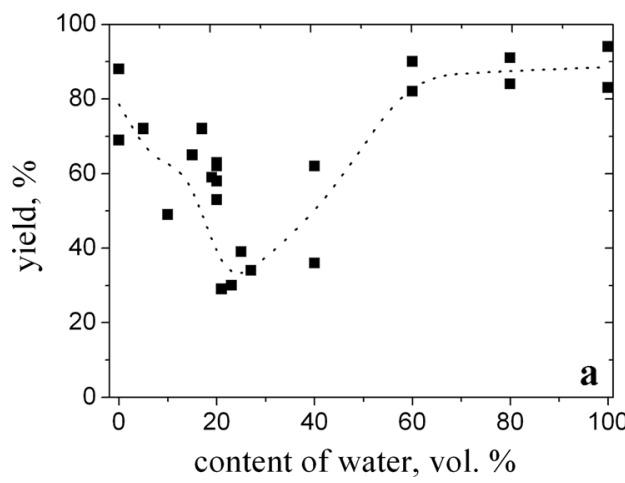

Fig. 3 a Dependence of yields of the polymers on water content in the solvent mixture for CA-AMPS polymerization in water-dioxane mixtures. b Dependence of intrinsic viscosity values of the polymers on water

very low; thus, high molecular mass samples have been prepared. The process as a whole takes few minutes only.

Polymerization of the surfmers in organic media

In contrast to their precursors AMPS and surfactants, salts of these compounds display rather good solubility in many organic solvents, which makes it possible to perform their polymerization in organic solutions. These polymerizations were conducted for $1 \mathrm{~h}$ in solvents differing in their chemical structure and polarity. The results are summarized in Table 1 .

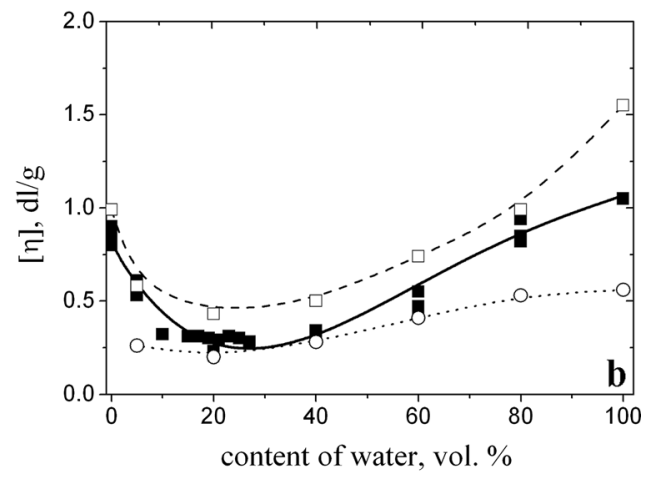

content in the solvent mixture for the surfmer polymerization in waterdioxane mixtures (empty square CA-AMPS, empty circle CTA-AMPS, filled square DDA-AMPS )

Polymerization degrees of the products evaluated by intrinsic viscosity values correlate with polarity of the solvents. Products with considerable values of intrinsic viscosity were obtained in those solvent (dioxane, chloroform), which could promote formation of reverse micelles. In general, the higher the polarity of the solvent, the lower is the polymerization degree of the products. Polymerization in dimethylformamide $(\varepsilon=38)$, which is not a micelle-forming solvent, results in formation of the products with intrinsic viscosity values below $0.10 \mathrm{dL} / \mathrm{g}$. Further increase of polarity leads to formation of direct micelles and increase of intrinsic viscosity

Table 1 Polymerization of the surfmers in different organic solvents

\begin{tabular}{|c|c|c|c|c|c|c|c|}
\hline Surfmer/solvent/concentration & $\varepsilon$ & $T\left({ }^{\circ} \mathrm{C}\right)$ & Initiator, $\mathrm{g} / \mathrm{L}$ & Yield, \% & {$[\eta]^{\mathrm{a}}, \mathrm{dL} / \mathrm{g}$} & $\mathrm{M} \eta \times 10^{-3}$ & Type of the product \\
\hline DDA-AMPS/dioxane/(0.25 M) & 2.2 & 60 & ACVA, 1 & 80 & 0.72 & $950^{\mathrm{b}}$ & Precipitate \\
\hline DDA-AMPS/CCl $/(0.10 \mathrm{M})$ & 2.2 & 70 & AIBN, 1 & 56 & 0.30 & $300^{\mathrm{b}}$ & Gel \\
\hline DDA-AMPS/p-xylene/(0.13 M) & 2.3 & 60 & AIBN, 2 & 80 & 0.40 & $440^{\mathrm{b}}$ & Gel \\
\hline DDA-AMPS/CHCl ${ }_{3} /(0.25 \mathrm{M})$ & 4.9 & 70 & AIBN, 1 & 92 & 0.60 & $750^{\mathrm{b}}$ & Solution \\
\hline DDA-AMPS/THF/(0.25 M) & 7.6 & 60 & AIBN, 1 & 88 & 0.27 & $260^{\mathrm{b}}$ & Solution $^{\mathrm{c}}$ \\
\hline CA-AMPS/dioxane/(0.25 M) & 2.2 & 60 & ACVA, 1 & 80 & 0.86 & & Precipitate \\
\hline CA-AMPS/CCl $/(0.10 \mathrm{M})$ & 2.2 & 70 & AIBN, 1 & 56 & 0.30 & & Gel \\
\hline CA-AMPS/ $p$-xylene $/(0.13 \mathrm{M})$ & 2.3 & 60 & AIBN, 2 & 80 & 0.40 & & Gel \\
\hline CA-AMPS/ $\mathrm{CHCl}_{3} /(0.25 \mathrm{M})$ & 4.9 & 70 & AIBN, 1 & 92 & 1.10 & & Solution \\
\hline CA-AMPS/THF/(0.25 M) & 7.6 & 60 & AIBN, 1 & 88 & 0.27 & & Solution $^{\mathrm{c}}$ \\
\hline CTA-AMPS/dioxane/(0.25 M) & 2.2 & 75 & CVA, 1 & 44 & 0.14 & & Precipitate \\
\hline CTA-AMPS $/ \mathrm{CCl}_{4} /(0.10 \mathrm{M})$ & 2.2 & 70 & AIBN, 1 & 26 & 0.13 & & Gel \\
\hline CTA-AMPS/p-xylene/(0.10 M) & 2.3 & 75 & AIBN, 2 & 35 & 0.21 & & Gel \\
\hline CTA-AMPS $/ \mathrm{CHCl}_{3} /(0.25 \mathrm{M})$ & 4.9 & 70 & AIBN, 4 & & 0.14 & & Solution \\
\hline CTA-AMPS/ethyl acetate $(0.1 \mathrm{M})$ & 6.0 & 70 & AIBN, 4 & 65 & 0.15 & & Precipitate \\
\hline
\end{tabular}

${ }^{\text {a }}$ Measured at $25^{\circ} \mathrm{C}$ in $\mathrm{CHCl}_{3}$

${ }^{\mathrm{b}}$ Calculated using the MKH equation $[\eta]=2.06 \times 10^{-5} \mathrm{M}^{0.76}$ [39]

${ }^{\mathrm{c}}$ Precipitation occurs upon cooling the reaction mixture 


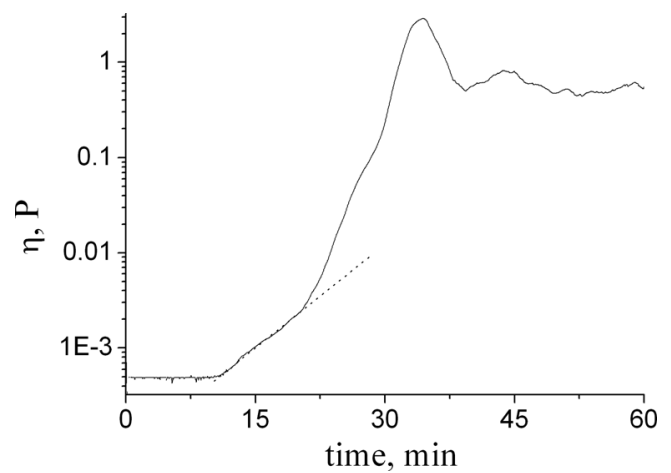

Fig. 4 Rheokinetic curve of polymerization of CA-AMPS in $p$-xylene

values of polymerization products. Of course, the polarity of the solvent is not the only factor influencing the results of the polymerization.

It should be noted that polymerization in tetrachloromethane and chloroform proceeds with formation of rather high molecular mass products, despite of their high chain transfer constants. We assume that the high local concentration of polymerizable groups in the reverse micelles promotes a high propagation rate and, on the contrary, lowers the rate of chain transfer reactions.

Results of polymerization of CA-AMPS and DDA-AMPS in $p$-xylene and tetrachloromethane are of special interest. Both products form tough, transparent, opalescent thermally irreversible gels in these solvents. Cavicchi, previously, only reported gel formation during RAFT polymerization of counterion surfmers [30].

Polymerization of CA-AMPS in $p$-xylene was conducted with real-time viscosity measurements. Rheokinetic curve is given in Fig. 4.

The polymerization proceeds with large inductive time, probably due to the presence of oxygen traces in the open measuring cell. After the beginning, it proceeds very fast, which is typical for micellar polymerization. After $25 \mathrm{~min}$ of the process (at $2800 \mathrm{mPa} \mathrm{s}^{-1}$ ), the gel breaks off the measuring plates of the viscometer.
We assume that the polymerization proceeds in reverse micelle solution. It correlates with AFM image of the particles adsorbed on mica from the solution of CAAMPS in $p$-xylene (Fig. 5a).

Currently, we do not have sufficient data to explain the mechanism of gel formation in the polymerization of the counterion surfmers in low-polarity solvents.

Studying and characterization of the polymers

The polymers synthesized in water and water-dioxane mixtures were main objects of the investigations. The polymers do not dissolve in water. Moreover, CAPAMPS does not even swell in $2 \mathrm{M} \mathrm{NaOH}$ and $2 \mathrm{M}$ $\mathrm{HCl}$ water solutions; DDA-PAMPS swells in these solutions a little, whereas CTA-PAMPS dissolves easily in both acid and base. The polymers dissolve in alcohols and in chloroform. Polymers display a straight plot of reduced viscosity on concentration in chloroform solutions and a weak polyelectrolyte effect in methanol, which indicates some dissociation. This fact enables us to "disassemble" DDA-PAMPS in $1 \mathrm{M} \mathrm{NaOH}$ in methanol and isolate PAMPS-Na by precipitating it in diethyl ether to measure its molecular mass. Walker used similar procedure to determine the molecular mass of CTAPVB [25]. Intrinsic viscosity values of the PAMPS-Na samples were measured in $1 \mathrm{M} \mathrm{NaCl}$, which suppresses a polyelectrolyte effect, and the molecular mass of the sample was evaluated using the Mark-Kuhn-Houwink (MKH) equation from [40]. In such manner, molecular masses of some DDA-PAMPS samples were calculated and $K$ and $\alpha$ of the MKH equation were obtained. $K=$ $2.1 \times 10^{-5} ; \alpha=0.78$. Very close values of the coefficients were obtained using independent diffusion and sedimentation methods $[\eta]=2.06 \times 10^{-5} \mathrm{M}^{0.76}$ (chloroform; $\mathrm{DMSO}+\mathrm{LiCl}$ ) [39]. Kuhn segment length (KSL) of DDA-PAMPS determined in the latter reference was found to be about $5 \mathrm{~nm}$, which is close to KSL of typical comb-like polymers.
Fig. 5 a AFM image of CAAMPS reverse micelles in $p$-xylene $(\mathrm{CM}=0.25 \mathrm{M})$. b Dependence of reduced viscosity of CAAMPS solution in $p$-xylene on concentration at $75^{\circ} \mathrm{C}$
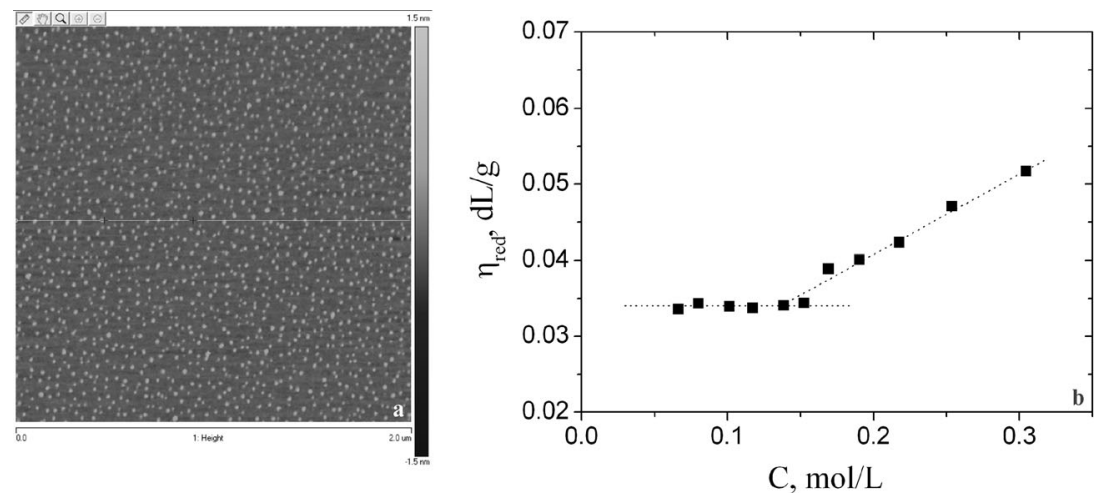

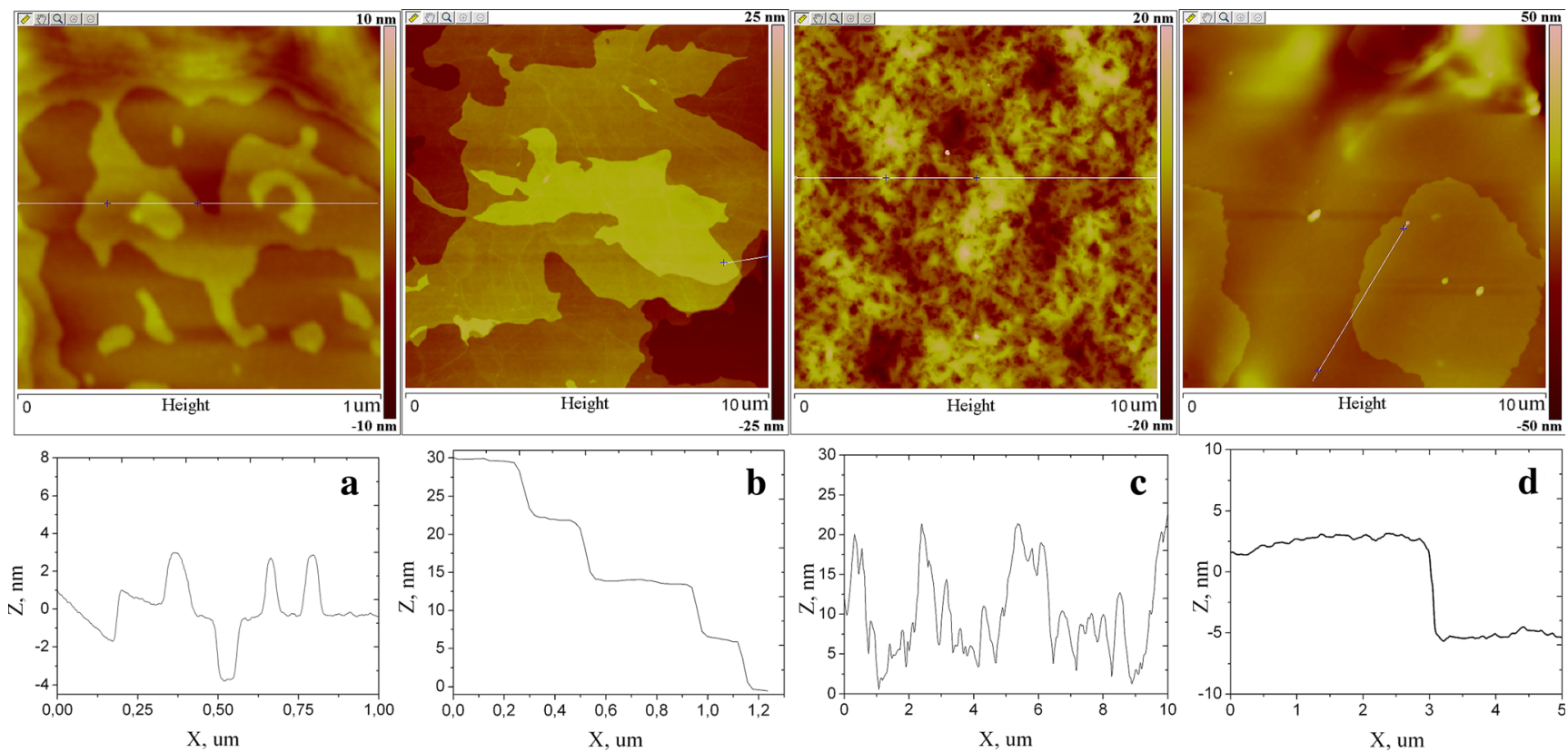

Fig. 6 AFM images of the polymer films. a Film of DDA-PAMPS from $\mathrm{CHCl}_{3}(C=1 \mathrm{~g} / \mathrm{L})$ adsorbed on a mica surface. b Free film of DDA-PAMPS from $\mathrm{CHCl}_{3}(C=20 \mathrm{~g} / \mathrm{L})$. c Free film of DDA-PAMPS from $\mathrm{CH}_{3} \mathrm{OH}(C=20 \mathrm{~g} / \mathrm{L})$. d Free film of CA-PAMPS from $\mathrm{CHCl}_{3}(C=20 \mathrm{~g} / \mathrm{L})$

AFM and SEM study of the PSC samples

Samples of DDA-PAMPS were investigated by AFM in a form of thin films prepared by solvent drying on a mica surface. Morphology of the films was found to be dependent strongly on the nature of a solvent. Films of DDA-PAMPS prepared from methanol solutions do not display explicit morphology. The films prepared from chloroform solutions aged during 3 days display distinct lamellar morphology (Fig. 6). Other polymers display quite similar behavior.

Morphology and structure of PSC prepared in different ways may differ considerably. We have studied by
Fig. 7 SEM images of the asprepared samples of the DDAPAMPS, synthesized in different media: a dioxane, $\mathbf{b}$ waterdioxane 20:80 mixture, $\mathbf{c}$ water, and $\mathbf{d} p$-xylene
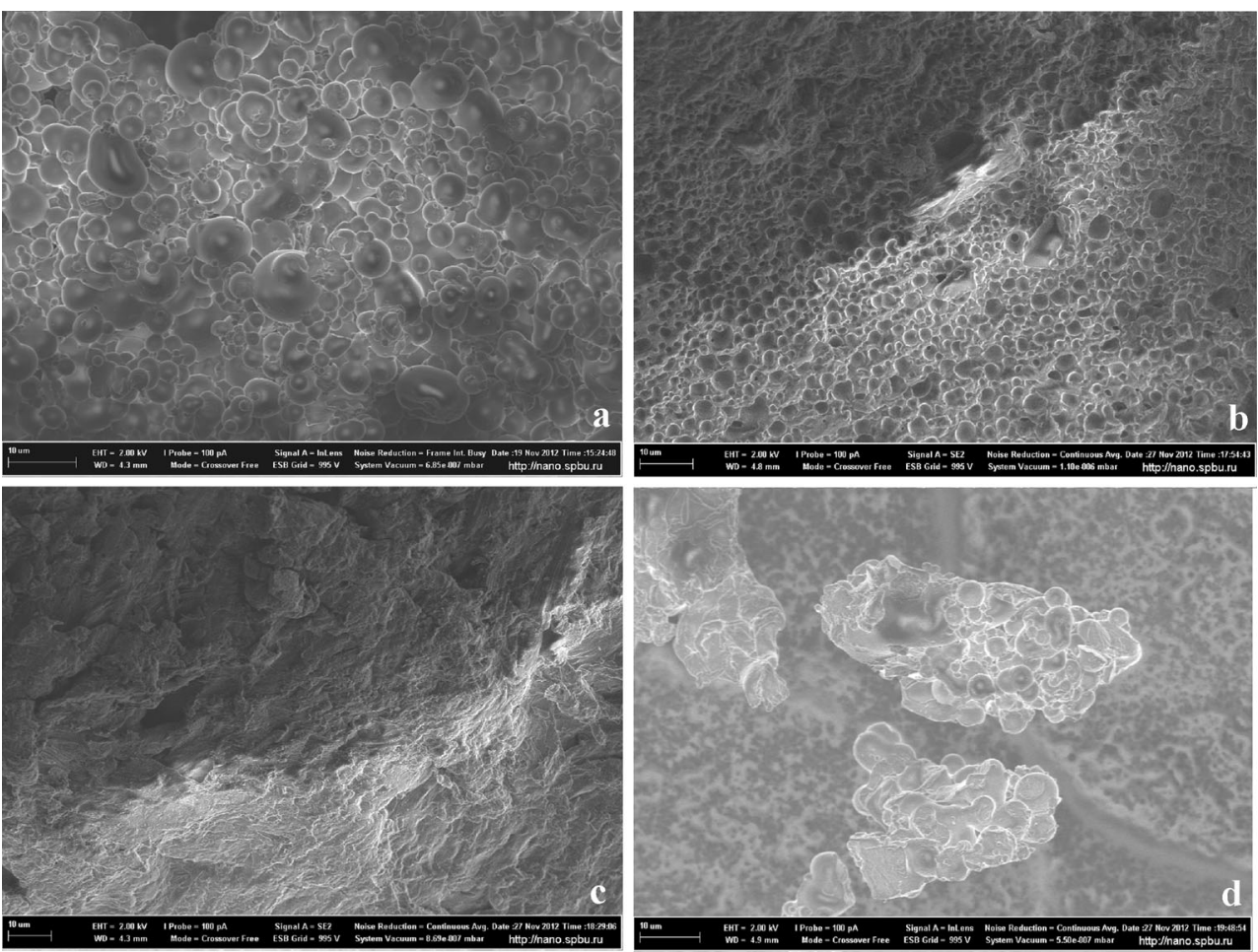
SEM some samples of DDA-PAMPS prepared by polymerization in different solvents and two samples prepared in traditional way by pouring together solutions of PAMPS and DDA.

Figure $7 \mathrm{a}-\mathrm{d}$ displays the morphology of the aspolymerized samples of DDA-PAMPS prepared in different solvent systems.

Really, the images of different samples differ greatly. One can see spherical particles with a diameter range of $1-5 \mu \mathrm{m}$ in Fig. 7a. This sample was polymerized with precipitation in dioxane in reverse micelle solution. Figure $7 \mathrm{~b}$ displays the shape of particles of the sample precipitated during polymerization in dioxane with $20 \%$ of water. The particles are rather unformed, although some of them retain signs of spherical shape. It is possible to see pieces of layered fragments. Figure $7 \mathrm{c}$ displays the shape of the particles precipitated from water solution during polymerization. There are no spherical particles in this sample. It is possible to identify extended bundles and plates. Figure $7 d$ displays the spherical particle from the powder prepared by extraction of the DDA-PAMPS gel polymerized in $p$-xylene. Thus, we can assume that the gel is spherically structured microparticles.

Thus, it is possible to compile some preliminary conclusions. Polymerization in nonpolar solvents (in reverse micelle solutions) leads to formation of spherically shaped particles of either precipitate or gel in the case of polymerization DDAAMPS in $p$-xylene. On increase of polarity of the polymerization media (dioxane with $20 \%$ water) morphology of the precipitated changes from spherical to distorted porous semispherical morphology, some signs of extended lamellar segments appear. Further increase of the polarity (pure water) changes the morphology completely to extended bundles and lamellas.

SAX diffraction (Fig. 8) confirms formation of lamellar structure of the product at polymerization in water

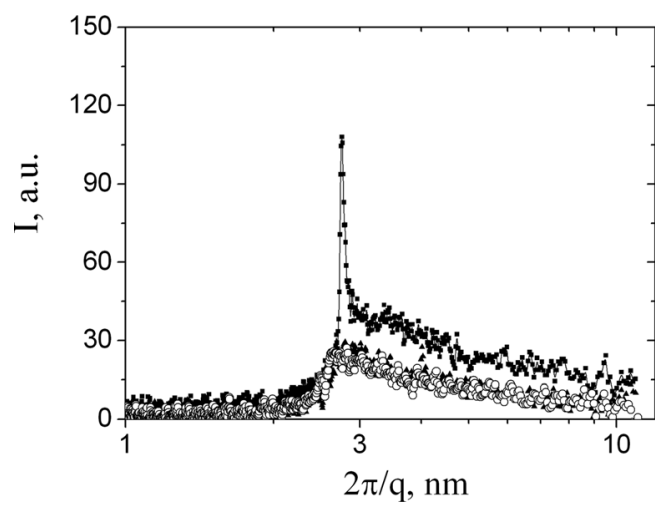

Fig. 8 SAX diffraction patterns for as-polymerized DDA-PAMPS samples, synthesized in different media: dioxane (triangles), water (squares), and water-dioxane 20:80 mixture (empty circles) and its absence in the samples prepared by polymerization in pure dioxane and dioxane-water 80:20 mixture.

This difference in morphology gives some grounds for preliminary considerations of the polymerization processes in various polymerization media. Polymerization in polar solvents begins in direct micelles with highly polar periphery, which determines interchain interactions with formation of layered structures with extended chains. Polymerization in nonpolar solvents begins in reverse micelles with low-polarity periphery. The polymerized chains when reaching a critical oligomer degree of polymerization for precipitation separate from the reaction media as paraffin-like nano-droplets at $60{ }^{\circ} \mathrm{C}$, which agglomerate to form solid spherical particles with $1-5 \mu \mathrm{m}$ in diameter. The proposed scheme is hypothetical and requires to be studied in more detail.

\section{Conclusion}

Summarizing the results concerning structure-property relationships of the polyalkylamino 2-acryl-2-methyl propane sulfonates, the following features should be mentioned:

- The highest molecular mass and yields were obtained for CA-PAMPS whereas the lowest for CTA-PAMPS. This fact does not follow the tendency, according to which has the higher hydrophobicity, the higher precipitation rate during polymerization, and the higher polymerization degrees.

- Solubility of the PSCs in water systems in wide $\mathrm{pH}$ range does not follow their hydrophobicity too; CTA-PAMPS, seeming to be most hydrophobic, dissolves in basic and acidic water solutions, whereas DDA-PAMPS and CAPAMPS do not.

These contradictions can be explained by differences in the nature of the ionic bond primary and quaternary amine with a sulfonic acid group. Ionic bonds of DDA-PAMPS and CAPAMPS represent tight ionic pair with strong attractive force between the charges due to small ionic radius of primary amino group (about $0.15 \mathrm{~nm}$ ). These bonds are tightly wrapped up by the hydrophobic shell, which creates a lowpolar environment around them, thereby making them "more covalent," and prevents a direct contact of the bonds with polar solvent. Ionic bond of CTA-PAMPS is much looser, because its ionic radius is about twice longer $(0.322 \mathrm{~nm})$. Therefore, molecules of polar solvent and ions of acids or bases can easily penetrate into this bond and solvate it.

The same reasons explain the difference and contradictions in the behavior of the monomers discussed in [32]. 
Acknowledgments The authors are grateful for the financial support of Russian Foundation for Basic Researches (grants nos. 12-03-00746, 1303-00474 and 15-03-08690). SEM images were obtained in the Interdisciplinary Resource Center for Nanotechnology. SAXD data were obtained in the Resource Center for Methods of Analysis of Composite Materials of St. Petersburg State University.

\section{References}

1. Goddard ED, Ananthapadmanabhan KP (eds) (1993) Interaction of surfactants with polymers and proteins. CRC, Boca Raton

2. Antonietti M, Conrad J, Thünemann A (1994) Polyelectrolytesurfactant complexes: a new type of solid, mesomorphous material. Macromolecules 27:6007-6011

3. Thünemann AF (2002) Polyelectrolyte-surfactant complexes (synthesis, structure and materials aspects). Prog Polym Sci 27:14731572

4. Ober CK, Wegner G (1997) Polyelectrolyte-surfactant complexes in the solid state: facile building blocks for self-organizing materials. Adv Mater 9:17-31

5. Chiappisi L, Hoffmann I, Gradzielski M (2013) Complexes of oppositely charged polyelectrolytes and surfactants - recent developments in the field of biologically derived polyelectrolytes. Soft Matter 9: 3896-3909

6. Dias RS, Magno LM, Valente AJM, Das D, Das PK, Maiti S, Miguel MG, Lindman B (2008) Interaction between DNA and cationic surfactants: effect of DNA conformation and surfactant headgroup. J Phys Chem B112:14446-14452

7. Verma A, Verma A (2013) Polyelectrolyte complex —an overview. IJPSR 4:1684-1691

8. Liu J-Y, Wang J-G, Li N, Zhou H-J, Sun P-C, Chen T-H (2012) Polyelectrolyte-surfactant complexes as a template for the synthesis of zeolites with intracrystalline mesopores. Langmuir 28:8600-8607

9. Buchhammer H-M, Lunkwitz GPK (2000) Nanoparticles based on polyelectrolyte complexes: effect of structure and net charge on the sorption capability for solved organic molecules. Colloid Polym Sci 278:841-847

10. Chang Y-M, Zhu R, Richard E, Chen C-C, Li G, Yang Y (2012) Electrostatic self-assembly conjugated polyelectrolyte surfactant complex as an interlayer for high performance polymer solar cells. Adv Funct Mater 22:3284-3289

11. Thünemann AF, Müller M, Dautzenberg H, Joanny J-F, Löwen H (2004) Polyelectrolyte complexes. Adv Polym Sci 166:113-171

12. Kotz J, Kosmella S, Beitz T (2001) Self-assembled polyelectrolyte systems. Prog Polym Sci 26:1199-1232

13. Kosmella S, Koltz J, Shirahama K, Liu J (1998) Cooperative nature of complex formation in mixed polyelectrolyte-surfactant systems. J Phys Chem B 102:6459-6464

14. Nause RG, Hoagland DA, Strey HH (2008) Structural evolution of complexes of poly(styrenesulfonate) and cetyltrimethylammonium chloride. Macromolecules 41:4012-4019

15. Fundin J, Brown W (1994) Polymer/surfactant interactions. Sodium poly(styrenesulfonate) and CTAB complex formation. Light scattering measurements in dilute aqueous solution. Macromolecules 27: 5024-5031

16. Ren B, Tong Z, Gao F, Liu X, Zeng F (2001) Fluorescence and X-ray diffraction studies on binding and complex of surfactants and dansylated polyelectrolytes with sulfonate groups. Polymer 42 : 7921-7928

17. Tong Z, Wang C, Ren B, Lin X, Zeng F (2003) Binding of ionic surfactants on oppositely charged polyelectrolytes observed by fluorescent methods. Chin J Polym Sci 21:609-620
18. Jain N, Trabelsi S, Guillot S, McLoughlin D, Langevin D, Pierre Letellier P, Turmine M (2004) Critical aggregation concentration in mixed solutions of anionic polyelectrolytes and cationic surfactants. Langmuir 20:8496-8503

19. Nizri G, Makarsky A, Magdassi S, Talmon Y (2009) Nanostructures formed by self-assembly of negatively charged polymer and cationic surfactants. Langmuir 25:1980-1985

20. von Ferber C, L wen H (2005) Polyelectrolyte-surfactant complex: phases of self-assembled structures. Faraday Discuss 128:389-405

21. Hentze H-P, Khrenov V, Tauer K (2002) A new approach towards redispersable polyelectrolyte-surfactant complex nanoparticles. Colloid Polym Sci 280:1021-1026

22. Piculell L, Norrman J, Svensson AV, Lynch I, Bernardes JS, Loh W (2009) Ionic surfactants with polymeric counterions. Adv Colloid Interface Sci 147-148:228-236

23. Lerebours B, Perly B, Pileni MP (1989) Polymerization of cetyltrimethylammonium methacrylate in direct micelles. Prog Colloid Polymer Sci 79:239-243

24. Hartman PC, Sanderson RD (2005) Templating polymerization of dodecylammonium surfactant with polymerizable (meth)acrylate counterion. Macromol Symp 225:229-237

25. Gerber MJ, Kline RS, Walker LM (2004) Characterization of rod-like aggregates generated from a cationic surfactant and a polymerizable counterion. Langmuir 20:8510-8516

26. Gerber MJ, Walker LM (2006) Controlling dimensions of polymerized micelles: micelle template versus reaction conditions. Langmuir 22:941-948

27. Kuntz DM, Walker LM (2007) Solution behavior of rod-like polyelectrolyte-surfactant aggregates polymerized from wormlike micelles. J Phys Chem B 111:6417-6424

28. Shulevich YV, Petzold G, Navrotskii AV, Novakov IA (2012) Properties of polyelectrolyte-surfactant complexes obtained by polymerization of an ionic monomer in a solution of an oppositely charged surfactant. Colloids Surfaces A: Physicochem Eng Aspects 415:148-152

29. Liu Y, Pollock KL, Cavicchi KA (2009) Synthesis of poly(trioctylammonium $p$-styrenesulfonate) homopolymers and block copolymers by RAFT polymerization. Polymer 50:62166217

30. Liu Y, Lloyd A, Guzman G, Cavicchi KA (2011) Polyelectrolytesurfactant complexes as thermoreversible organogelators. Macromolecules 44:8622-8630

31. Bilibin AY, Sukhanova TM, Matuschkin NI, Mel'nikov AB, Zorin IM (2012) Polymerization of dodecylammonium-2-acrylamido-2methylpropane sulfonate in solvents with different dielectric constants and study of the resulting ionic complexes. Macromol Symp 317-318:160-168

32. Bilibin AYu, Sukhanova TM, Kondratenko YuA, Zorin IM (2013) nAlkyl ammonium 2-acrylamido-2-methylpropanesulfonates: Synthesis, properties, and polymerization Polymer Science Series B $55 \mathrm{p} 22-30$

33. Odian G (2004) Principles of polymerization, 4th edn. Wiley, Hoboken, pp 297-298

34. Bai F, Yang X, Li R, Huang B, Huang W (2006) Monodisperse hydrophilic polymer microspheres having carboxylic acid groups prepared by distillation precipitation polymerization. Polymer 47: $5775-5784$

35. Li GL, M hwald H, Shchukin DG (2013) Precipitation polymerization for fabrication of complex core-shell hybrid particles and hollow structures. Chem Soc Rev 42:3628-3646

36. Slomkowski S, Alemán J, Gilbert RG et al (2011) Terminology of polymers and polymerization processes in dispersed systems (IUPAC Recommendations 2011). Pure Appl Chem 83:2246 
37. Liu T, De Simone JM, Roberts GW (2006) Kinetics of the precipitation polymerization of acrylic acid in supercritical carbon dioxide: the locus of polymerization. Chem Eng Sci 61:3129-3139

38. Tajima K, Aida T (2000) Controlled polymerizations with constrained geometries. Chem Commun 2399-2412

39. Andreeva LN, Shcherbinina TM, Zorin IM, Bezrukova MA, Bushin SV, Bilibin AY (2013) Molecular, conformational, and optical characteristics of poly(dodecylammonium-2-acrylamido-2methylpropanesulfonate) in organic solvents. Polymer Sci Ser A 55:289-294

40. Fischer LW, Sochor AR, Tan JS (1977) Chain characteristics of poly(2-acrylamido-2-methylpropanesulfonate) polymers. 1. Light scattering and intrinsic viscosity studies. Macromolecules 10:949954 